Research Paper

\title{
Andrographolide Inhibits Angiogenesis by Inhibiting the Mir-21-5p/TIMP3 Signaling Pathway
}

Jianwei Dai ${ }^{1 *}$, Yuyin Lin ${ }^{1 *}$, Youfa Duan ${ }^{2 *}$, Zixuan Li², Dalei Zhou ${ }^{2}$, Wensheng Chen ${ }^{1}$, Lijing Wang ${ }^{2 \otimes}$ and Qian-Qian Zhang ${ }^{2 \bowtie}$

1. GMU-GIBH Joint School of Life Sciences, Guangzhou Medical University, Guangzhou 510000, China;

2. Vascular Biology Research Institute, School of Basic Course, Guangdong Pharmaceutical University, Guangzhou 510006, China.

* These authors contributed equally to this work.

$\triangle$ Corresponding authors: Qian-Qian Zhang (Guangdong Pharmaceutical University, Waihuan Road East \#280, Guangzhou, Guangdong 510006, PR China. Tel: 86-20-39352397, E-mail: zhangqianqian@gdpu.edu.cn) and Lijing Wang (Guangdong Pharmaceutical University, Waihuan Road East \#280, Guangzhou, Guangdong 510006, PR China. Tel: 86-20-39352231, E-mail: wanglijing62@163.com).

(c) Ivyspring International Publisher. This is an open access article distributed under the terms of the Creative Commons Attribution (CC BY-NC) license (https://creativecommons.org/licenses/by-nc/4.0/). See http://ivyspring.com/terms for full terms and conditions.

Received: 2017.01.15; Accepted: 2017.03.17; Published: 2017.05.16

\begin{abstract}
Angiogenesis provides nutrients and oxygen to promote tumor growth and affords a channel that facilitates tumor cell entry into the circulation. Andrographolide (Andro) possess anti-tumor activity; however, its direct effect on angiogenesis still needs to be clarified. In this study, our experiments revealed that Andro significantly inhibited vascular growth in chick embryo chorioallantoic membrane (CAM) and yolk sac membrane (YSM) models. Meanwhile, tumor angiogenesis was also suppressed by Andro. Additionally, we found that cell proliferation, migration and tube formation of vascular endothelial cells was inhibited by Andro treatment in vitro. The effect was primarily mediated through inhibition of miR-21-5p expression and further targeting of TIMP3. This work provides evidence that Andro directly inhibits angiogenesis and might be an effective anti-angiogenic therapeutic drug for cancer treatment.
\end{abstract}

Key words: Andrographolide; angiogenesis; miR-21-5p; TIMP3.

\section{Introduction}

Angiogenesis is a process of endothelial cells proliferation, breakdown of the extracellular matrix (ECM), migration of endothelial cells and formation of new capillaries [1]. Tumor angiogenesis supports tumor growth and metastasis by providing essential nutrients and oxygen and providing a route for tumor cells to enter the circulation [2, 3]. Therefore, anti-angiogenesis is thought to be an important therapeutic approach for treating cancer.

Many molecules have been shown to be involved in regulating angiogenesis, including vascular endothelial growth factor (VEGF) and its receptor VEGFR, basic fibroblast growth factor (bFGF), matrix metalloproteinases (MMPs) and tissue inhibitors of metalloproteinases (TIMPs). Several VEGF/VEGFR-targeting drugs are now used in the clinic to treat cancer. MicroRNAs (miRNAs) are a class of small noncoding RNAs that regulate protein expression at the posttranscriptional level. Recently, some evidence has indicated that miRNAs might contribute to the regulation of angiogenesis [4-6]. Therefore, miRNA-based drugs that target angiogenesis will likely be developed in the future.

miR-21-5p is an important oncogenic miRNA that is often overexpressed in various solid tumors and its regulatory mechanism is well characterized. miR-21-5p could promote tumor growth and metastasis by targeting several tumor suppressors including PTEN, PDCD4, Bcl2 and TIMP3 [7-10]. TIMP3 inhibits angiogenesis by blocking the binding of VEGF and VEGF receptor-2 [11]. Therefore, miR-21-5p might be involved in regulating angiogenesis. Recently, it has been reported that miR-21-5 $p$ induced angiogenesis by activating AKT and ERK and HIF-1a/VEGF/VEGFR2-associated signaling pathway [12, 13]. However, whether 
miR-21-5p is involved in Andro-mediated inhibition of angiogenesis is largely undetermined.

The extracellular matrix (ECM) is an important controlling factor that modulates angiogenesis. Degradation of the capillary basement membrane by matrix metalloproteinases (MMPs) is the initiating stage of angiogenesis. Therefore, MMPs are inhibited by the ECM-bound protein TIMP3 and play an important role in regulating angiogenesis. Nevertheless, whether TIMP3 is associated with Andro-induced inhibition of angiogenesis requires further clarification.

Andrographolide (Andro) is a labdane diterpenoid isolated from the stem and leaves of Andrographis paniculata, a Chinese herbal medicine [14]. It has been demonstrated that Andro possess various anti-inflammatory and anticancer activities. We previously reported that Andro can inhibit tumor growth in insulinoma and melanoma by inactivating the NF-KB signaling pathway $[15,16]$. Additionally, we found that microvascular density was decreased in tumor tissues. A decrease IN angiogenesis is beneficial for the inhibition of tumor growth and metastasis. Recently, it has been reported that Andro can inhibit angiogenesis by targeting MMP2 and MMP9 or by blocking VEGFA/VEGFR2-MAPK signaling cascade signals $[17,18]$. Angiogenesis is a complex regulatory network; however, whether other signals might also be involved in Andro-induced inhibition of angiogenesis needs to be studied further.

In this study, we used a chick embryo models and human umbilical vein vascular endothelial cells to define the possible role and molecular mechanism of Andro in tumor angiogenesis.

\section{Materials and methods}

\section{Reagents and Antibodies}

Andrographolide (Andro, Sigma-Aldrich) was dissolved in dimethyl sulfoxide (DMSO). miRNA mimics of miR-21-5p and negative control RNA (NC) were obtained from RiboBio Co., Ltd. The following primary antibodies were used for immunoblotting: rabbit anti-TIMP3 (ab39184), obtained from Abcam (Cambridge, UK); and rabbit anti-GAPDH (\#2118), obtained from Cell Signaling Technology, Inc. (Danvers, Massachusetts, USA).

\section{Cell Lines and Transfection}

The 293T cell line and MDA-MB-231 breast cancer cell line were obtained from the cell bank of Chinese Academy of Sciences (Shanghai, China) and maintained in Dulbecco's modified Eagle's medium (DMEM, Gibco) supplemented with $10 \%$ fetal bovine serum (FBS), $100 \mathrm{U} / \mathrm{mL}$ penicillin and $100 \mu \mathrm{g} / \mathrm{mL}$ streptomycin. Human umbilical vein vascular endothelium cells (HUVECs) were isolated from human umbilical cord vein as described previously [19]. All the cells were incubated at $37^{\circ} \mathrm{C}$ in a humidified chamber supplemented with $5 \% \mathrm{CO}_{2}$. Transfection of HUVECs with miR-21-5p mimics was performed using Lipofectamine 3000 (Invitrogen) at a final concentration of $100 \mathrm{nM}$.

\section{Chick embryo chorioallantoic membrane (CAM) assay}

Fertilized chicken eggs were purchased from the Avian Farm of South China Agriculture University (Guangzhou, China). The eggs shells were cleaned with $75 \%$ ethanol and incubated for 9 days at a temperature of $37 \pm 1^{\circ} \mathrm{C}$ and relative humidity of $50 \%-60 \%$. Then, the shell was cut and removed to create a small window $(1 \mathrm{~cm} \times 1 \mathrm{~cm})$ above the air chamber. Andro $(5 \mu \mathrm{M}, 10 \mu \mathrm{M}$ or $20 \mu \mathrm{M})$ or DMSO was added to the air chamber, and then, the eggs were sealed with sterile medical tape and incubated for 48 $\mathrm{h}$. The CAM vasculature was further assessed under a microscope (Olympus SZX16). Vessel density was recorded as the percentage of blood vessel area in the whole area compared with the DMSO group using an Image-Pro Plus 6.0 analysis system.

\section{Chick embryo yolk sac membrane (YSM) assay}

Fertilized chicken eggs were incubated for 3 days and then cracked into a sterile dish. The blood vessels of the yolk sac were faced upward in the dish, and two silastic rings, with an inside diameter of $9.5 \mathrm{~mm}$ and an outside diameter of $11 \mathrm{~mm}$, were placed on the top of the vessel regions. Then, $40 \mu \mathrm{L}$ of Andro $(5 \mu \mathrm{M}$, $10 \mu \mathrm{M}$ or $20 \mu \mathrm{M}$ ) or DMSO was introduced into the rings on the top of the well-developed vessels. The morphology of the vessels within the rings was photographed under a stereomicroscope (Olympus SZX16) using an OPTPRO 2007 image acquisition system at $0 \mathrm{~h}, 12 \mathrm{~h}$ and $24 \mathrm{~h}$. The area of the blood vessels occupied was quantified using an Image-Pro Plus 6.0 analysis system.

\section{Experimental Breast cancer Assay}

To further evaluate the effect of Andro on tumor angiogenesis, MDA-MB-231 cells were cultured on the CAM model as previously reported [20]. Fertilized chicken eggs were prepared for the CAM assay and incubated for 10 days. Then, the silastic rings were placed on the CAM, and $5 \times 10^{6}$ MDA-MB-231 cells in $20 \mu \mathrm{L}$ of PBS were added to the middle of the rings and incubated for $48 \mathrm{~h}$. Andro $(5 \mu \mathrm{M}, 10 \mu \mathrm{M}$ or $20 \mu \mathrm{M})$ or DMSO was added to the center of the rings for $48 \mathrm{~h}$. The tumor size and vasculature were further assessed under a stereomicroscope (Olympus SZX16). The length (L) and width (W) of the tumors were measured, the tumor volume was calculated as 
$\left(\mathrm{L} \times \mathrm{W}^{2}\right) \times 0.5236$, and vessel density was recorded as described [16]. The tumors were fixed with $4 \%$ paraformaldehyde and embedded in paraffin for further examination.

\section{Histological and immunoblotting analysis}

The tumor tissues on the CAM were fixed in $4 \%$ paraformaldehyde for $24 \mathrm{~h}$. Then, the tissues were embedded in paraffin and cut into 4- $\mu \mathrm{m}$-thick sections. The sections were stained with H\&E for routine histological analysis. The total protein in cells was prepared using RIPA buffer and further analyzed. All the procedures were performed as previously described [21].

\section{Cell Viability Assay}

HUVECs (1500 cells/well) were plated in a 96-well plate, and the indicated concentration of Andro was added $24 \mathrm{~h}$ later. After $48 \mathrm{~h}, \mathrm{CCK} 8$ reagent was added to the wells and the plate was incubated for $3 \mathrm{~h}$ at $37^{\circ} \mathrm{C}$ and then measured at $570 \mathrm{~nm}$.

\section{In vitro HUVEC Tube Formation Assay}

A 96-well plate was coated with Matrigel for $2 \mathrm{~h}$. HUVECs in endothelial cell growth medium (EGM, CC-3162, Lonza) supplement with Andro $(20 \mu \mathrm{M})$ or DMSO were added to the pre-coated plate at a density of $1.5 \times 10^{5}$ cells $/ \mathrm{mL}$ and maintained in a $37^{\circ} \mathrm{C}$ humidified incubator for $5 \mathrm{~h}$ to form vascular tubes. The tubes were photographed using an inverted light microscope.

\section{In vitro HUVEC Cell Migration Assay}

Transwell 24-well chambers $(8 \mu \mathrm{m}$ pore size, Costar) were precoated with Matrigel $(250 \mu \mathrm{g} / \mathrm{mL})$ dissolved in EGM without growth supplements. Andro- or DMSO- treated HUVECs were harvested and resuspended in EGM without growth supplements. Meanwhile, $20 \mu \mathrm{M}$ Andro or a corresponding dose of DMSO was added in the upper compartment of the chamber and placed in 24-well plates containing $0.6 \mathrm{~mL}$ of EGM with growth supplements. After a 12-h incubation, the upper surface of the chamber was wiped with a cotton tip and the lower surface was stained with crystal violet for $15 \mathrm{~min}$. The membranes were photographed using an inverted light microscope.

\section{Rat Aortic Ring Assay}

The thoracic aorta was isolated from 6-week-old male Sprague-Dawley rats (Guangdong Medical Laboratory Animal Center, China) and rinsed three times using ice-cold PBS. The aorta was cut into 1- to 1.5-mm-long segments and placed on a 48- well plate, which was coated with $100 \mu \mathrm{L}$ of cold Matrigel and maintained at $37^{\circ} \mathrm{C}$ in a humidified chamber supplemented with $5 \% \mathrm{CO}_{2}$ for $30 \mathrm{~min}$ to form a gel. Afterwards, the top of each of the aorta sections was covered with $100 \mu \mathrm{L}$ of the Matrigel and maintained for $30 \mathrm{~min}$ in a $37^{\circ} \mathrm{C}$ humidified incubator. EGM with Andro $(20 \mu \mathrm{M})$ or DMSO was added to the wells, and the aortic sections were cultured for 10 days before being photographed and analyzed.

\section{Quantitative real-time PCR}

Total RNA was extracted from HUVECs that were treated with Andro or DMSO using TRIzol reagent (Invitrogen) according to the manufacturer's instructions. Total RNA (500 ng) was reverse transcribed, and then, a real-time quantitative PCR analysis was performed as previously reported [21]. All the RT and PCR primers for miR-21-5p and U6 were obtained from RiboBio Co., Ltd. The data were analyzed using the $2-\Delta \triangle \mathrm{CT}$ methodology as previously described [22].

\section{Luciferase Assays}

Briefly, 293T cells $\left(5.5 \times 10^{4}\right.$ cells/well) were cultured in 48 -well plates. The reporter vectors, which included the TIMP3 3'UTR and target site mutant 3'UTR, were constructed and then cotransfected with the reporter vectors and the miRNA expression plasmid $24 \mathrm{~h}$ later. The luciferase activity was measured after $48 \mathrm{~h}$ using a Dual-Luciferase Reporter Assay System (Promega). The primers used to construct the reporter vectors were as follows: TIMP3 3'UTR Forward Primer 5'-CTCGAGCAAGGAGG AACTTGGGTG-3' and TIMP3 3'UTR Reverse Primer 5'-GCGGCCGCAATACAGAAGTGTCT-3'; TIMP3 target site mutant Forward Primer 5'-CTCGAGCAAGGAGGAACTTGGGTG-3' and TIMP3 target site mutant Reverse Primer 5'-GCGGCCGCAATACAGAAGTGTCT-3'.

\section{Statistical Analysis}

All data are presented as the mean \pm standard deviation (S.D.) of 3 separate experiments. The differences between two groups were analyzed using a Student's two-sided $t$-test. Differences were considered statistically significant if $P<0.05$. The intensity of the immunoblotting bands was quantified and normalized to GAPDH using Quantity One software (Bio-Rad, USA).

\section{Results}

\section{Andro inhibits angiogenesis in a chick embryo CAM model}

A chick embryo CAM model was employed to explore the direct anti-angiogenic effect of Andro. The CAM was treated with DMSO (control group) or 5 
$\mu \mathrm{M}, 10 \mu \mathrm{M}$ or $20 \mu \mathrm{M}$ Andro for $48 \mathrm{~h}$. The results showed that angiogenesis in the CAM was markedly inhibited by Andro in a dose-dependent manner compared with the DMSO group (Fig. 1A). Moreover, the growth of branches in the CAM blood vessels was also suppressed by Andro (Fig. 1B). Then, the MVD was quantitatively analyzed using IPP software, and the results also indicated that Andro suppressed the density of blood vessels in the chick embryo CAM model (Fig. 1C). All the results suggest that Andro can directly inhibit angiogenesis.

\section{Andro suppresses angiogenesis in a YSM model}

The chick embryo YSM model is another system that is suitable for the study of angiogenesis. Therefore, we employed this model to further confirm the direct role of Andro in angiogenesis. To better quantify the effect of Andro on angiogenesis, silastic rings were placed on the YSM and DMSO (control group) or $5 \mu \mathrm{M}, 10 \mu \mathrm{M}$ or $20 \mu \mathrm{M}$ Andro was added into the rings. The blood vessels in the rings were observed and photographed at $0 \mathrm{~h}, 12 \mathrm{~h}$ and $24 \mathrm{~h}$. Then, the blood vessel plexus growth and extension within the silastic rings was compared. The results revealed that Andro could also inhibit the extension of the blood vessel plexus in a dose- and time-dependent manner (Fig. 2A). Meanwhile, the statistical results showed that the blood vessel density was also reduced by Andro (Fig. 2B). This model further confirmed that Andro could directly inhibit angiogenesis.

\section{Andro inhibits tumor growth and tumor angiogenesis in a CAM model}

MDA-MB-231 cells were cultured on a 10-day-old chick embryo CAM to confirm the anti-angiogenic role of Andro in tumor progression. MDA-MB-231 cells were seeded in the silastic rings on the CAM and treated with DMSO (control group) or 5 $\mu \mathrm{M}, 10 \mu \mathrm{M}$ or $20 \mu \mathrm{M}$ Andro. As shown in Fig. 3A and $3 \mathrm{~B}$, the tumor volume was significantly reduced with Andro treatment, and the effect was dose dependent. Moreover, the blood vessel density was also suppressed by Andro compared with the DMSO group (Fig. 3C and 3D). Furthermore, the tumor tissues were peeled off and then fixed, embedded and stained with H\&E (Fig. 3E). The staining results revealed that multiple regions of neovascularization can be seen in the tumor tissues of the DMSO group, while Andro significantly attenuated the neovascularization in the tumor tissues in a dose-dependent manner. These results reveal that Andro can inhibit angiogenesis in tumor tissues during breast cancer progression.

A
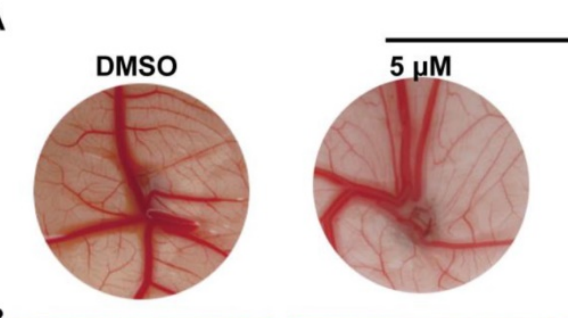

Andro
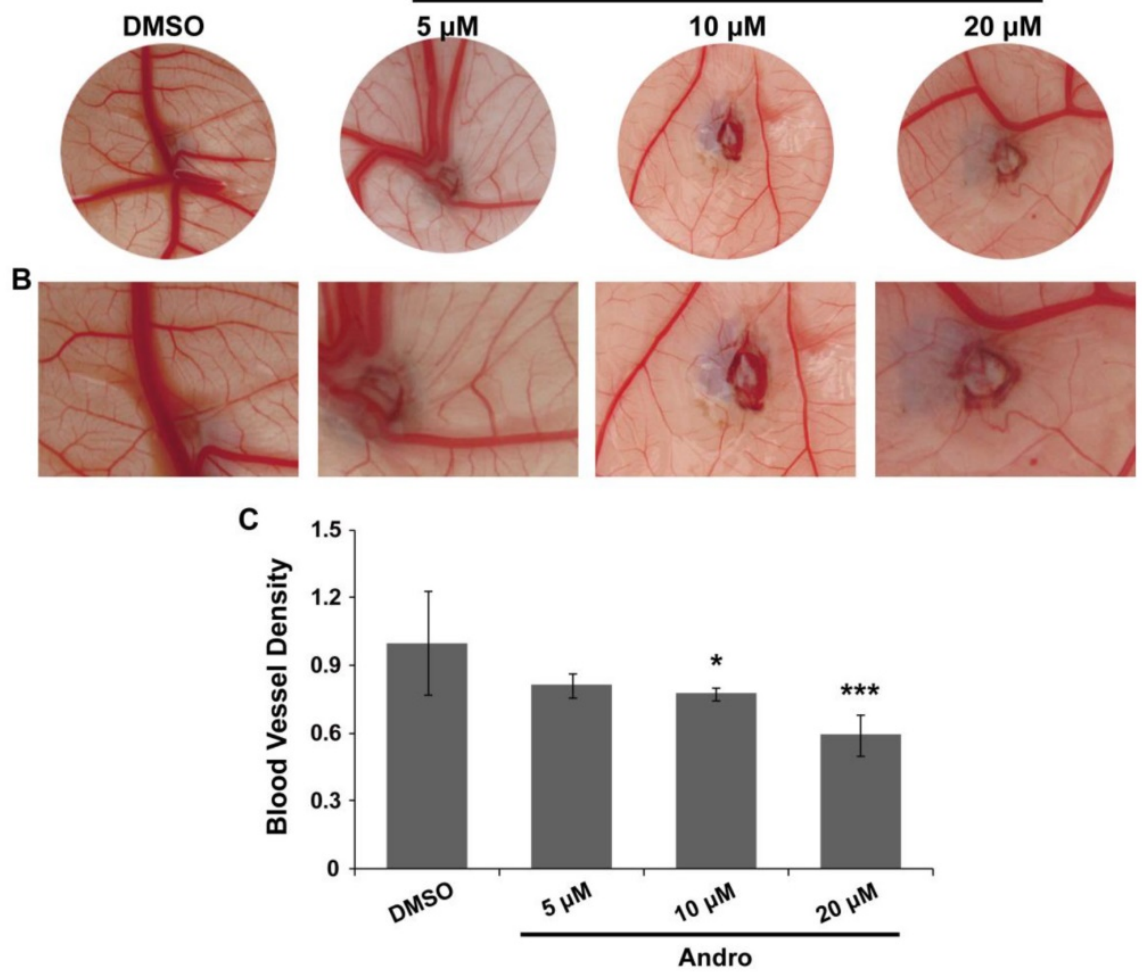

Figure 1. Andro inhibits angiogenesis in a chick embryo CAM model. A CAM assay was used to determine the effects of Andro on angiogenesis. (A) Representative images show the appearance of blood vessels in chick embryo CAMs treated with DMSO or $5 \mu M, 10 \mu M$ or $20 \mu M$ Andro for 24 h. (B) Higher magnification of A, showing the caliber of principal blood vessels; there were fewer blood vessel branches in the Andro-treated CAMs than in the DMSO group. (C) Statistical analysis of the CAMs showed that Andro significantly decreased blood vessel density (MVD). $* P<0.05, * * * P<0.001$. 




Figure 2. Andro inhibits angiogenesis in a chick embryo YSM model. YSM assays were used to detect the effect of Andro on angiogenesis. (A) The blood vessel plexus in chick embryo YSMs treated with DMSO or $5 \mu \mathrm{M}, 10 \mu \mathrm{M}$ or $20 \mu \mathrm{M}$ Andro at the start of the experiment or after $12 \mathrm{~h}$ and $24 \mathrm{~h}$. (B) Statistical analysis of the blood vessel density of Andro-treated YSMs compared with the DMSO group.

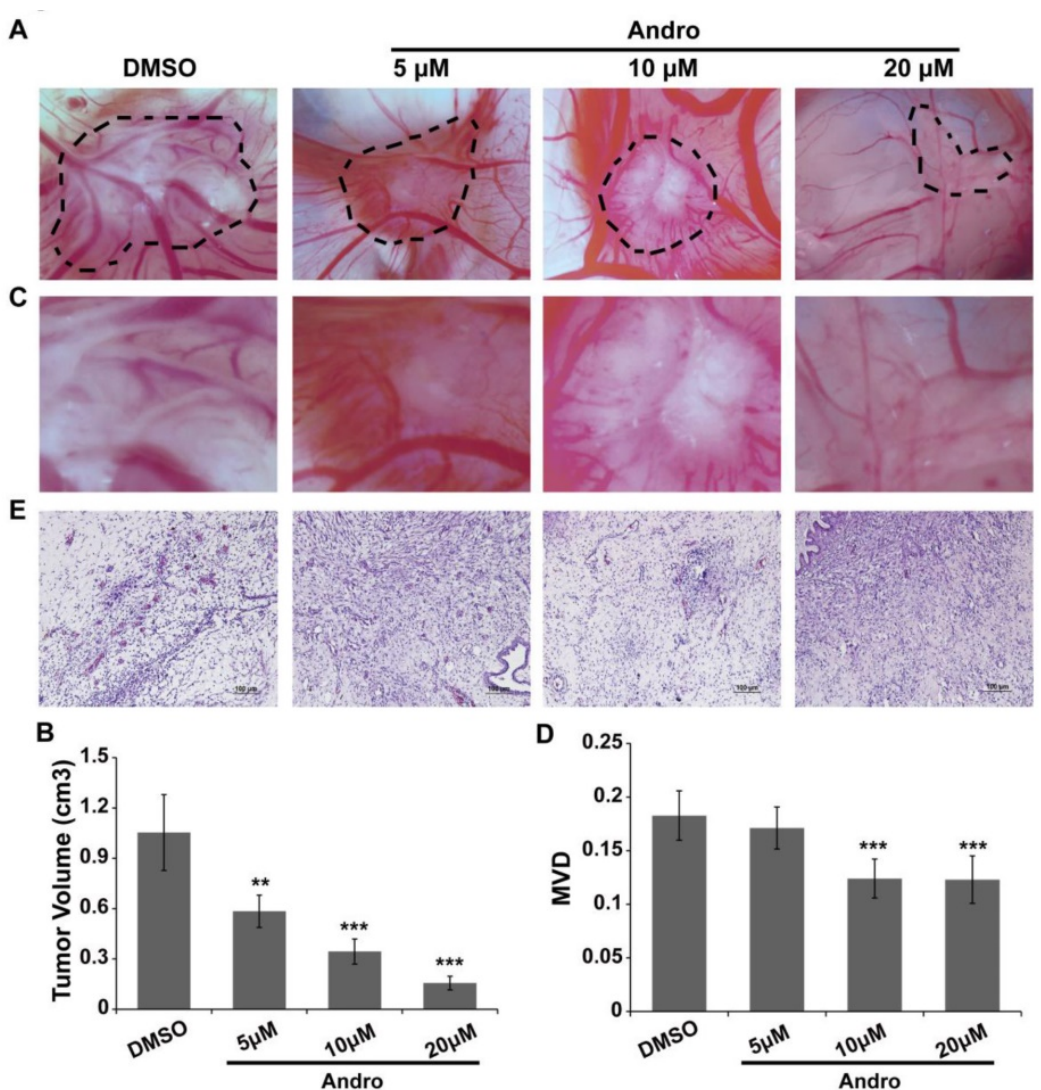

Figure 3. Andro inhibits tumor growth and angiogenesis in a chick embryo CAM model. MDA-MB-231 cells were implanted on the 10-day-old chick CAM and incubated for $48 \mathrm{~h}$. (A) Representative images show the appearance of breast cancer xenografts treated with DMSO or $5 \mu \mathrm{M}, 10 \mu \mathrm{M}$ or $20 \mu \mathrm{M}$ Andro. (B) Higher magnification of A, showing fewer blood vessel branches in Andro-treated CAMs than in the DMSO group. Statistical analysis of tumor volume (C) and MVD (D) of DMSO- and Andro-treated tumors that seed on CAM model. (E) H\&E staining of tumor tissues on CAMs. $* * P<0.01, * * * P<0.001$. 


\section{Andro decreases microvessel formation}

Andro inhibited angiogenesis in the CAM and YSM models; however, the regulatory mechanism still needed to be further elucidated. Tube formation and the migration of vessel epithelial cells are the hallmark of angiogenesis. Therefore, we explored the effect of Andro on vascular epithelial cells. First, the results showed that Andro could suppress cell viability in a dose-dependent manner and the $\mathrm{IC}_{50}$ was approximately $20 \mu \mathrm{M}$ (Fig. 4A). Then, we found that Andro could markedly restrain the migration of HUVECs (Fig. 4B). Furthermore, we determined the effect of Andro on HUVEC tube formation in vitro.
Compared with DMSO group, Andro-treated HUVECs could not form capillary-like microtubule networks (Fig. 4C). All the above data indicated that Andro could inhibit angiogenesis by regulating the viability, migration and tube formation ability of vessel epithelial cells. Furthermore, to detect the directly effect of Andro on microvessel formation, an ex vivo rat aortic rings assay was employed. The rat aorta was removed and cut into 1- to 1.5-mm-long segments and then put in Matrigel to let the epithelial cells form microvessels. As shown in Fig. 4D, Andro significantly reduced the outgrowth of epithelia cells to form microvessels.

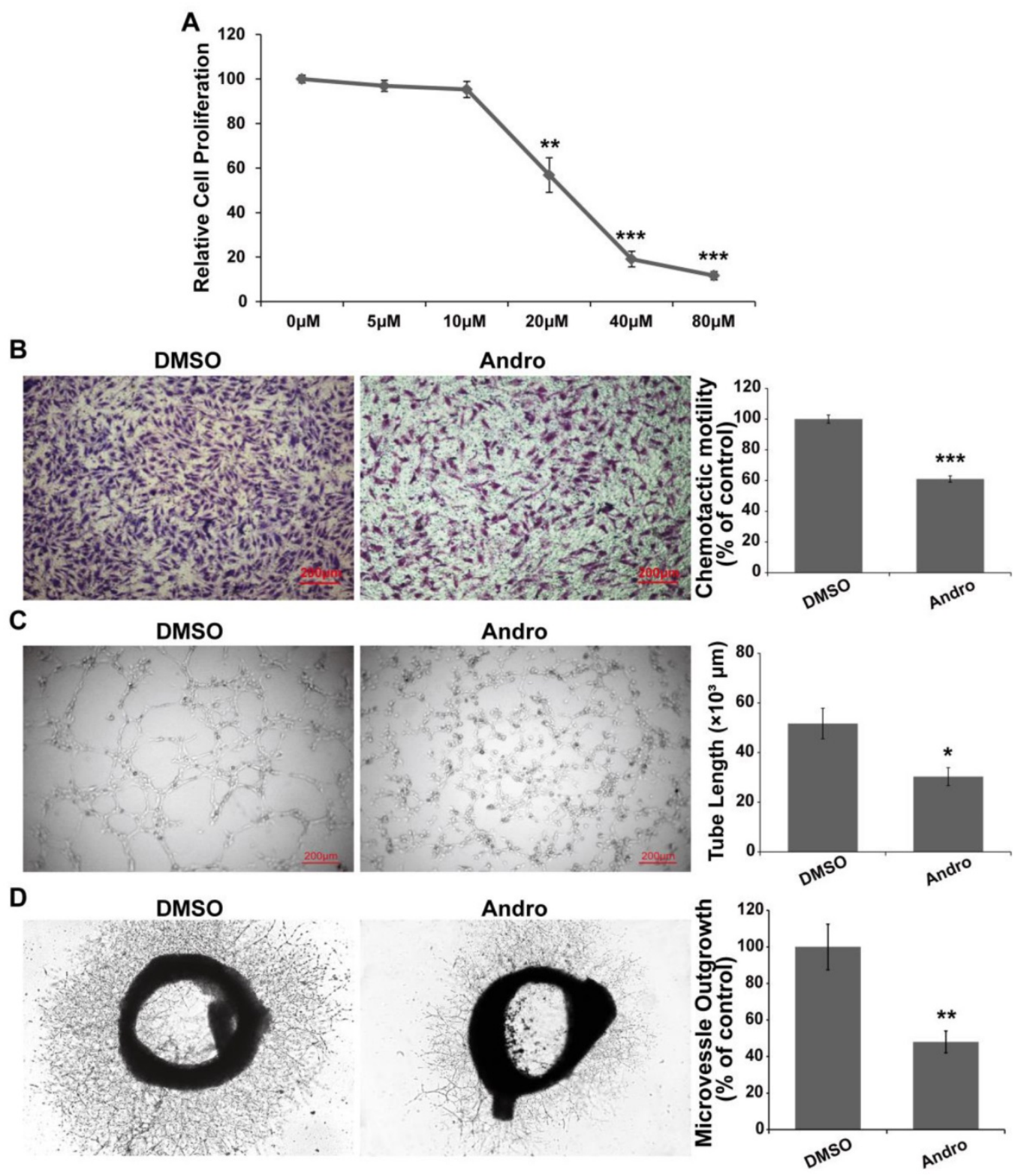

Figure 4. Andro suppresses vascular endothelial cell proliferation, migration and tube formation. (A) Andro significantly inhibited HUVEC proliferation in a dose-dependent manner in an MTT assay. (B) The migration ability of HUVECs was determined using Transwell assays. HUVECs were harvested and added to the upper chamber, and the cell migration was significantly inhibited by Andro. (C) HUVECs were cultured on Matrigel and treated with DMSO or $20 \mu M$ Andro, and the extension of tubes was inhibited by Andro. (D) A rat aortic ring assay was used to detect the effect of Andro on endothelial cell migration. The thoracic aorta was isolated and cut into 1-to 1.5 - $\mathrm{mm}$-long segments and then cultured in Matrigel and treated with $20 \mu \mathrm{M}$ Andro or DMSO for 10 days. Photographs of representative rings from assays performed in triplicate. 


\section{Andro inhibits angiogenesis by suppressing the expression of miR-21-5p}

Our previous data showed that Andro could inhibit tumor growth by suppressing the NF-kB signaling pathway. miR-21-5p is a target of NF- $\mathrm{kB}$ in regulating cell proliferation in solid tumor [23]. However, it has been shown that miR-21-5p is involved in angiogenesis [12, 24, 25]. To test whether
miR-21-5p is involved in the anti-angiogenic process of Andro, the expression of miR-21-5p was detected. Our results showed that Andro significantly inhibited miR-21-5p expression in HUVECs (Fig. 5A). In addition, miR-21-5p co-treated with Andro could reverse the effect of Andro on tube formation and migration of HUVECs (Fig. 5B and 5C). These data suggested that miR-21-5p is involved in angiogenesis.
A

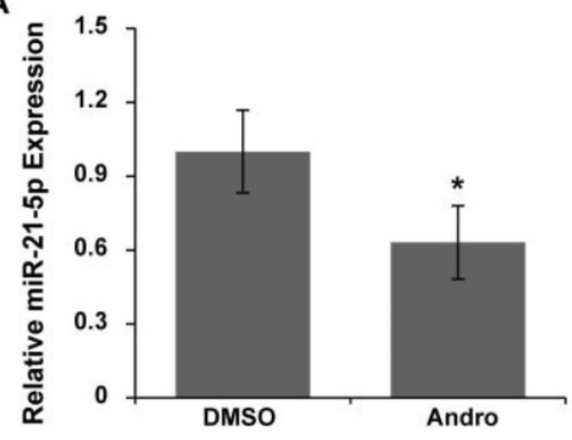

B
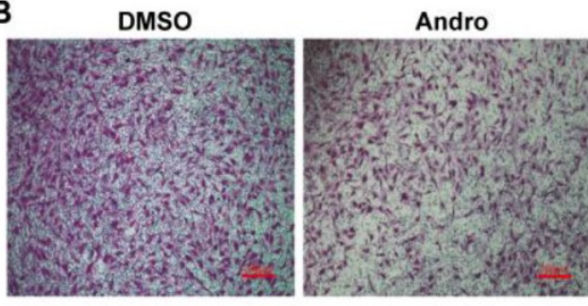

C


Hsa-miR-21-5p 3'

TIMP3 site mutation $\quad$ ' '...GGUUUUGAGCUUUCUCGCCATCG...

Hsa-miR-21-5p

I I I I I I I

AGUUGUAGUCAGACUAUUCGAU



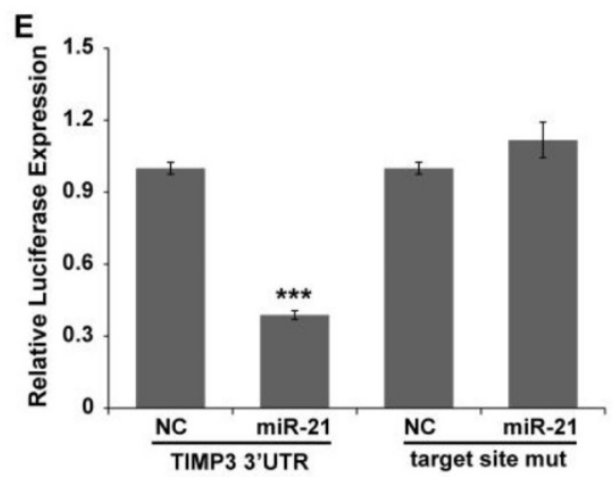

$\mathbf{F}$

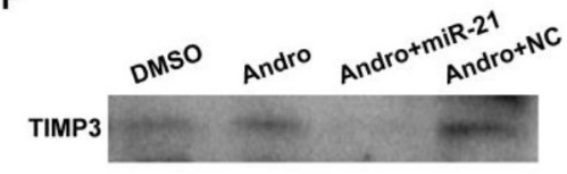

GAPDH

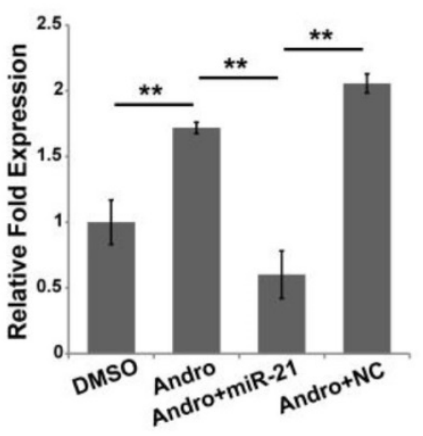

Figure 5. miR-21-5p is involved in the anti-angiogenic activity of Andro. (A) The expression of miR-21-5p was significantly inhibited by Andro compared with the DMSO group, determined using quantitative real-time PCR. (B) The migration ability of HUVECs was detected using Transwell assays. HUVECs were transfected with NC or miR-21-5p and then harvested and added to the upper chamber. Cell migration was significantly inhibited by Andro, and the inhibition effect was reversed by miR-21-5p. (C) The transfected HUVECs were cultured on Matrigel and treated with DMSO or $20 \mu M$ Andro. The extension of tubes was inhibited by Andro, and the effect was abolished by miR-21-5p. (D) TIMP3 possesses a binding site for miR-21-5p in the 3'UTR and mutations were introduced into the seed sequences complementary to the 5' end of miR-21-3p. (E) Luciferase assays indicated that miR-21-5p directly down-regulated the expression of TIMP3. Relative luciferase values were normalized to those of the group cotransfected with NC. Renilla luciferase activity was normalized to firefly luciferase expression. The mean \pm standard deviation from 5 separate determinations. (E) Western blotting showed that the expression of TIMP3 in HUVECs was inhibited by Andro, and the effect was abolished by overexpression of miR-21-5p. GAPDH served as an internal control. *** $P<0.001$; ns indicates $P>0.05$. 
A recent report has indicated that miR-21-5p promotes angiogenesis by targeting TIMP3/MMPs signaling [26]. A reporter vector and mutant vector were constructed (Fig. 5D). A luciferase reporter assay was performed to determine whether miR-21-5p directly bind to the TIMP3 3'UTR. The results indicated that miR-21-5p significantly inhibited the expression of the Renilla luciferase reporter that carried the wild-type target site of TIMP3 compared with the control group, and the effect was reversed when mutations were introduced into the seed sequence complementary to miR-21-5p (Fig. 5E). In addition, Andro promoted the expression of TIMP3 protein in HUVECs compared with DMSO-treated cells. Nevertheless, the effect of Andro could be reversed by co-treated with Andro and miR-21-5p in HUVECs (Fig. 5F). Our results showed that Andro can inhibit angiogenesis by targeting miR-21-5p and regulating TIMP3.

\section{Discussion}

This study clearly demonstrated an anti-angiogenic role of Andro during tumorigenesis. Our findings support a model in which Andro directly inhibits the expression of miR-21, which then targets TIMP3 and suppresses the expression of MMP9, thereby inhibiting cell growth, migration and tube formation of vascular endothelial cells.

Tumor growth is dependent on the availability of nutrients and oxygen. Initially, the growth of a tumor is supported by nearby blood vessels [27]. However, the tumor volume is restricted only by the consumption of nutrients and oxygen [28]. The formation of new blood vessels is necessary for tumor progression. Angiogenesis not only supplies the necessary nutrients and oxygen for tumor cells but also provides a route for tumor cell metastasis to target organs. Recently, anti-angiogenic drugs have emerged as a new class of therapeutic compounds to treat cancer. Many products have been developed and are used in the clinic to destroy tumor vasculature, including bevacizumab, pegaptanib, anecortave acetate, ovalicin and TNP-470. However, a report has indicated that some anti-angiogenic drugs that can effectively inhibit tumor angiogenesis in certain cancers show less clinical benefit in other cancers [29]. Therefore, anti-angiogenic therapy is usually combined with conventional chemotherapy drugs to treat cancer. Another possibility approach is to find an effective drug that inhibits both angiogenesis and tumor cell growth and metastasis to treat cancer.

Andrographolide is a labdane diterpenoid and the major bioactive constituent derived from the medicinal plant Andrographis paniculata. It is used for anti-inflammatory and anti-infective therapy in the clinic. Andro has been found to have anti-cancer effects in various solid tumors, by inhibiting cell proliferation and invasion, inducing cell apoptosis, stimulating the production of cytotoxic $\mathrm{T}$ lymphocytes and suppressing angiogenesis [18, 30-33]. Our previous work combined with the findings in this study have demonstrated that Andro possesses anti-cancer activity through targeting multiple pathways, including inhibiting cell proliferation and invasion, induceing cell apoptosis and decreasing angiogenesis. Therefore, Andro may potentially be used as a multi-target anti-tumor drug for clinical therapy.

The chick chorioallantoic membrane (CAM) is an extremely rich vascular membrane from 4- to 14-day-old chick embryos and the yolk sac membrane (YSM) is the blood vessel network that develops into arteries and veins on days 3 to 5 of chick embryo development $[34,35]$. Both the CAM and YSM are well established models for studying angiogenesis or screening anti-angiogenic drugs. An experimental in vivo tumor model is a model in which tumor cells grow on the chick CAM for 2 days [20]. Moreover, only the tumor cells are of human origin, and the blood vessels in the tumor are newly formed during tumorigenesis in this model. Due to the low costs, short experimental duration and ease of manipulation, this model makes it possible to quickly screen anti-angiogenic drugs. In this study, we used these chick embryo models to clarify the anti-angiogenic effect of Andro.

miRNAs are a class of short non-coding RNAs that post-transcriptionally regulate gene expression. It has been demonstrated that the abnormal expression of miRNAs can regulate tumor growth and development. Currently, accumulating evidence has indicated that miRNAs can regulate tumor angiogenesis by targeting angiogenic factors and protein kinases. miR-21-5p has been identified as an oncogene that is often overexpressed in a wide variety of solid tumor tissues [36]. In addition, miR-21-5p has been found to induce angiogenesis by targeting multiple signaling pathways $[12,37,38]$. However, we still do not know whether miRNA is involved in the anti-angiogenic activity of Andro. In this work, we are the first to demonstrate that miR-21-5p can promote angiogenesis and that this effect may be inhibited by Andro during tumorigenesis.

In summary, our data have demonstrated that Andro can function as an anti-angiogenic drug by targeting the miR-21-5p regulatory network, and it may be used in the clinic to improve outcomes for cancer patients due to the anti-proliferative, anti-metastatic, anti-angiogenic and pro-apoptotic therapeutic potential. 


\section{Abbreviations}

Andro: Andrographolide; miRNA: microRNA; HUVEC: Human Umbilical Vein Vascular Endothelium cell; H\&E: hematoxylin \&eosin; MVD: Microvessel density; CAM: chorioallantoic membrane; YSM: yolk sac membrane; ECM: extracellular matrix; VEGF: vascular endothelial growth factor; bFGF: basic fibroblast growth factor; MMP: matrix metalloproteinase; TIMP: tissue inhibitor of metalloproteinase.

\section{Acknowledgments}

The authors thank Dr. Cuiling Qi and Dr. Lingyun Zheng for technical assistance.

\section{Funding}

This research was supported by the National Natural Science Foundation of China (31471290), the Natural Science Foundation of Guangdong Province, China (2014A030313582 and 2016A030313601), the Science and Technology Planning Project of Guangdong Province, China (2013B021800082 and 2015A030302083), the Science and Technology Foundation of Guangzhou, China (201510010076), Pearl River S\&T Nova Program of Guangzhou, China (201610010045) and Training Program for Excellent Young Teachers in Guangdong Province, China (YQ2015100).

\section{Competing Interests}

The authors have declared that no competing interest exists.

\section{References}

1. Gao Z, Zheng J, Yang B, Wang Z, Fan H, Lv Y, et al. Sonodynamic therapy inhibits angiogenesis and tumor growth in a xenograft mouse model. Cancer letters. 2013; 335: 93-99.

2. Folkman J, Shing Y. Angiogenesis. The Journal of biological chemistry. 1992; 267: 10931-10934.

3. Liotta LA, Kleinerman J, Saidel GM. Quantitative relationships of intravascular tumor cells, tumor vessels, and pulmonary metastases following tumor implantation. Cancer research. 1974; 34: 997-1004.

4. Poliseno L, Tuccoli A, Mariani L, Evangelista M, Citti L, Woods K, et al. MicroRNAs modulate the angiogenic properties of HUVECs. Blood. 2006; 108: 3068-3071.

5. Yang WJ, Yang DD, Na S, Sandusky GE, Zhang Q, Zhao G. Dicer is required for embryonic angiogenesis during mouse development. The Journal of biological chemistry. 2005; 280: 9330-9335.

6. Sen CK, and Chan YCC. MicroRNA Regulation of Angiogenesis. Angiogenesis and Vascularisation. Springer Vienna. 2013: 187-212.

7. Si ML, Zhu S, Wu H, Lu Z, Wu F, Mo YY. miR-21-mediated tumor growth. Oncogene. 2007; 26: 2799-2803.

8. Asangani IA, Rasheed SA, Nikolova DA, Leupold JH, Colburn NH, Post S, et al. MicroRNA-21 (miR-21) post-transcriptionally downregulates tumor suppressor Pdcd4 and stimulates invasion, intravasation and metastasis in colorectal cancer. Oncogene. 2008; 27: 2128-2136.

9. Meng F, Henson R, Wehbe-Janek H, Ghoshal K, Jacob ST, Patel T. MicroRNA-21 regulates expression of the PTEN tumor suppressor gene in human hepatocellular cancer. Gastroenterology. 2007; 133: 647-658.

10. Gabriely G, Wurdinger T, Kesari S, Esau CC, Burchard J, Linsley PS, et al. MicroRNA 21 promotes glioma invasion by targeting matrix metalloproteinase regulators. Mol Cell Biol. 2008; 28: 5369-5380.

11. Qi JH, Ebrahem Q, Moore N, Murphy G, Claesson-Welsh L, Bond M, et al. A novel function for tissue inhibitor of metalloproteinases-3 (TIMP3): inhibition of angiogenesis by blockage of VEGF binding to VEGF receptor-2. Nature medicine. 2003; 9: 407-415.
12. Liu LZ, Li C, Chen Q, Jing Y, Carpenter R, Jiang $Y$, et al. MiR-21 induced angiogenesis through AKT and ERK activation and HIF-1alpha expression. PloS one. 2011; 6: e19139.

13. Zhao $\mathrm{D}, \mathrm{Tu} \mathrm{Y}$, Wan $\mathrm{L}, \mathrm{Bu} \mathrm{L}$, Huang $\mathrm{T}$, Sun $\mathrm{X}$, et al. In vivo monitoring of angiogenesis inhibition via down-regulation of mir-21 in a VEGFR2-luc murine breast cancer model using bioluminescent imaging. PloS one. 2013; 8: e71472.

14. Chakravarti RN, Chakravarti D. Andrographolide, the active constituent of Andrographis paniculata Nees; a preliminary communication. Indian medical gazette. 1951; 86: 96-97.

15. Zhang QQ, Ding Y, Lei $Y$, Qi CL, He XD, Lan T, et al. Andrographolide suppress tumor growth by inhibiting TLR4/NF-kappaB signaling activation in insulinoma. International journal of biological sciences. 2014; 10: 404-414.

16. Zhang OQ, Zhou DL, Ding Y, Liu HY, Lei Y, Fang HY, et al. Andrographolide inhibits melanoma tumor growth by inactivating the TLR4/NF-kappaB signaling pathway. Melanoma research. 2014; 24: 545-555.

17. Pratheeshkumar P, Kuttan G. Andrographolide inhibits human umbilical vein endothelial cell invasion and migration by regulating MMP-2 and MMP-9 during angiogenesis. J Environ Pathol Toxicol Oncol. 2011; 30: 33-41.

18. Shen K, Ji L, Lu B, Xu C, Gong C, Morahan G, et al. Andrographolide inhibits tumor angiogenesis via blocking VEGFA/VEGFR2-MAPKs signaling cascade. Chemico-biological interactions. 2014; 218: 99-106.

19. Marin V, Kaplanski G, Gres S, Farnarier C, Bongrand P. Endothelial cell culture: protocol to obtain and cultivate human umbilical endothelial cells. J Immunol Methods. 2001; 254: 183-190.

20. Hagedorn M, Javerzat S, Gilges D, Meyre A, de Lafarge B, Eichmann A, et al. Accessing key steps of human tumor progression in vivo by using an avian embryo model. Proc Natl Acad Sci U S A. 2005; 102: 1643-1648.

21. Xu H, He JH, Xiao ZD, Zhang QQ, Chen YQ, Zhou H, et al. Liver-enriched transcription factors regulate microRNA-122 that targets CUTL1 during liver development. Hepatology. 2010; 52: 1431-1442.

22. Schmittgen TD, Livak KJ. Analyzing real-time PCR data by the comparative C(T) method. Nat Protoc. 2008; 3: 1101-1108.

23. Shin VY, Jin $\mathrm{H}, \mathrm{Ng}$ EK, Cheng AS, Chong WW, Wong CY, et al. NF-kappaB targets miR-16 and miR-21 in gastric cancer: involvement of prostaglandin E receptors. Carcinogenesis. 2011; 32: 240-245.

24. Liu Y, Luo F, Wang B, Li H, Xu Y, Liu X, et al. STAT3-regulated exosomal miR-21 promotes angiogenesis and is involved in neoplastic processes of transformed human bronchial epithelial cells. Cancer letters. 2016; 370: 125-135.

25. Jiang FS, Tian SS, Lu JJ, Ding XH, Qian CD, Ding B, et al. Cardamonin Regulates miR-21 Expression and Suppresses Angiogenesis Induced by Vascular Endothelial Growth Factor. BioMed research international. 2015; 2015: 501581.

26. Hu J, Ni S, Cao Y, Zhang T, Wu T, Yin X, et al. The Angiogenic Effect of microRNA-21 Targeting TIMP3 through the Regulation of MMP2 and MMP9. PloS one. 2016; 11: e0149537.

27. Hanahan D, Weinberg RA. Hallmarks of cancer: the next generation. Cell 2011; 144: 646-674

28. Hippe A, Homey B, Mueller-Homey A. Chemokines. Recent results in cancer research Fortschritte der Krebsforschung Progres dans les recherches sur le cancer. 2010; 180: 35-50.

29. Moserle L, Jimenez-Valerio G, Casanovas O. Antiangiogenic therapies: going beyond their limits. Cancer discovery. 2014; 4: 31-41.

30. Sheeja K, Kuttan G. Activation of cytotoxic T lymphocyte responses and attenuation of tumor growth in vivo by Andrographis paniculata extract and andrographolide. Immunopharmacology and immunotoxicology. 2007; 29: 81-93.

31. Geethangili M, Rao YK, Fang SH, Tzeng YM. Cytotoxic constituents from Andrographis paniculata induce cell cycle arrest in jurkat cells. Phytotherapy research : PTR. 2008; 22: 1336-1341.

32. Pratheeshkumar P, Sheeja K, Kuttan G. Andrographolide induces apoptosis in B16F-10 melanoma cells by inhibiting NF-kappaB-mediated bcl-2 activation and modulating p53-induced caspase- 3 gene expression. Immunopharmacology and immunotoxicology. 2012; 34: 143-151.

33. Zhai Z, Qu X, Li H, Ouyang Z, Yan W, Liu G, et al. Inhibition of MDA-MB-231 breast cancer cell migration and invasion activity by andrographolide via suppression of nuclear factor-kappaB-dependent matrix metalloproteinase-9 expression. Molecular medicine reports. 2015; 11: 1139-1145.

34. Ausprunk DH, Knighton DR, Folkman J. Differentiation of vascular endothelium in the chick chorioallantois: a structural and autoradiographic study. Developmental biology. 1974; 38: 237-248.

35. le Noble F, Moyon D, Pardanaud L, Yuan L, Djonov V, Matthijsen R, et al. Flow regulates arterial-venous differentiation in the chick embryo yolk sac. Development. 2004; 131: 361-375.

36. Yao $\mathrm{O}, \mathrm{Xu} \mathrm{H}$, Zhang $\mathrm{OQ}$ Zhou $\mathrm{H}, \mathrm{Qu}$ LH. MicroRNA-21 promotes cell proliferation and down-regulates the expression of programmed cell death 4 (PDCD4) in HeLa cervical carcinoma cells. Biochemical and biophysical research communications. 2009; 388: 539-542.

37. Zhao Y, Xu Y, Luo F, Xu W, Wang B, Pang Y, et al. Angiogenesis, mediated by miR-21, is involved arsenite-induced carcinogenesis. Toxicology letters. 2013; 223: $35-41$

38. $\mathrm{Xu} X$, Song $\mathrm{N}$, Zhang $\mathrm{X}$, Jiao $\mathrm{X}, \mathrm{Hu}$ J, Liang $\mathrm{M}$, et al. Renal protection mediated by hypoxia inducible factor-1alpha depends on pro-angiogenesis function of miR-21 by targeting thrombospondin 1 . Transplantation. 2016 . 